\title{
ASD Children Gait Classification Based On Principal Component Analysis and Linear Discriminant Analysis
}

\author{
Nur Khalidah Zakaria ${ }^{1}$, Nooritawati Md Tahir ${ }^{1,2, *}$ Rozita Jailani $^{1}$ \\ ${ }^{1}$ Faculty of Electrical Engineering, Universiti Teknologi MARA (UiTM), 40450 Shah Alam, Selangor, Malaysia. \\ ${ }^{2}$ Advanced Analytics Engineering Centre (AAEC), Universiti Teknologi MARA (UiTM), 40450 Shah Alam, \\ Selangor, Malaysia. \\ *Corresponding Author: nooritawati@ieee.org
}

\begin{abstract}
The aim of this study is to explore the potential of the markerless-based gait features using both unsupervised and supervised algorithms namely the Principal Component Analysis (PCA) and Linear Discriminant Analysis (LDA) as feature extraction techniques. Firstly, a depth camera that created the three-dimensional (3D) skeleton image of the subject upon detection of movements by the motion sensor is used as data acquisition device in acquiring the walking gait of $30 \mathrm{TD}$ and $23 \mathrm{ASD}$ group. Next, the extracted gait features are translated into two categories named as Direct Joint Feature (DIR) and Reference Joint Feature (REF) features. To evaluate the effectiveness of PCA and LDA as feature extraction, Support Vector Machine (SVM), Naïve Bayes Classifier (NBC) and Artificial Neural Network (ANN) are chosen as classifiers. Overall results showed that LDA is the most suitable feature extraction with accuracy of 99.33\% using NBC as classifier with the DIR feature dataset as inputs.
\end{abstract}

Key words: Artificial Neural Network, Linear Discriminant Analysis, markerless-based gait technique, Naïve Bayesian, Principal Component Analysis, Support Vector Machine.

\section{INTRODUCTION}

Autism Spectrum Disorder (ASD) is a development disorders that can be characterized by difficulties in learning process, communication and social skill [1]. The symptoms are typically recognized in early childhood, for instance as early as two years [2] \& [3] and can be associated with developmental deficit. There are several methods use for screening ASD for instance via questionnaires that will normally assess parents or guardian reports. In addition, tools such as Childhood Autism Rating Scale (CARS), Gilliam Autism Rating Scale (GARS) and to name a few as mentioned in [4] are also used to diagnose ASD. This lifelong condition has no known cure [5], however this condition can be reduced and improved through therapies and treatments. Moreover, according to the Diagnostic and Statistical Manual of mental disorders (DSM-5), the existence of motor deficits that include abnormal gait, clumsiness and irregular motor signs are additional characteristics that support the diagnosis of ASD [1]. On that note, abnormal gait can be defined as existence of deviation from the normal walking gait. Since gait analysis can provide the details about the quantitative measurements of locomotion and movement, therefore deviations in motion can be used as one of the methods in analyzing pathological gait versus normal gait.

On the other hand, gait analysis and measurement is crucial in clinical applications, biomechanical analysis, computer graphics, and human identification. At present, most available measurement techniques for the ASD children are based on external markers or sensors that are attached to significant anatomical positions of the human body [6], [7] [8], [9], [10], [11] \& [12]. With marker and sensor techniques, gait measurement can provide precise motion information however, such techniques required intrusive specialized hardware and subject's contact. Throughout extensive searching in ASD gait classification and to the extent of our knowledge, only J. A. Vilensky et al. [13] used markerless-based technique to assess gait disturbance specifically related to ASD children. Hence, more study can be conducted to explore markerless techniques that will require less experimental protocol and ease of usage during experimental purpose so that it can be further used outside the laboratory environment specifically a new markerless method without having to use markers to be attached to the body that could lead to participants' maladjustment and anxiety [11].

Meanwhile, combination of several gait features were proven to be able to increase accuracy in classifying the autism walking gait pattern as reported in [8], [14] \& [15]. However, redundancy of information may contribute to inefficient machine learning process since the purpose of classification is to identify the most significant features prior to detection of gait pattern. Conversely, from previous researches, PCA was used to transform the waveform data into the respective PCs for the assessment of both magnitude and pattern differences across one gait cycle [16] and as reported in [17], PCA was used as feature selection. As discussed in [18], due to the high-dimensionality data, PCA is applied to the original raw data that resulted $98 \%$ PCs to be retained from the overall 
data and further acted as inputs to classifiers. Meanwhile in a model-free gait recognition, PCA and LDA is once again applied as feature extraction to the gait energy image (GEI) in extracting the main feature vectors of the gait [19]. Similarly, in gait kinematics classification, LDA is employed to improve the accuracy of recognition by projecting the data in the direction that reduced the intra-class variance whilst increased the inter-class variance [20].

Conversely, to evaluate the ability of PCA and LDA as feature extraction in classifying the gait feature between both ASD and TD children, the use of machine learning were implemented. As we know, machine learning algorithms is used for computational methods in learning the information directly from the data without relying on a predetermined equation and adaptively improve its performance as the number of data available during the learning process increased. Some well-known classifiers namely artificial neural network (ANN), support vector machine (SVM) and Naïve Bayes (NB) are employed for recognition and classification of broad areas in pathological gait such as in Parkinson's disease [21] \& [22], stroke patients [23], cerebral palsy [24], age-dependent gait [25] \& [26], persons with gait disorders [18] \& [27] as well as for recognition-based studies such as posture [28], walking [29] and fall detection [30].

On that note and based on the above research gap, this study deems further to propose a new approach for anomaly gait detection by classifying the walking gait in ASD children based on markerless-based approach with the depth camera and motion sensor as data acquisition device. Next, PCA and LDA will be evaluated as features extraction in identifying significant gait features. This study will mainly focus on extraction of potential gait features as well as optimization of classifier parameters during classification using three classifiers namely AAN, SVM and Naïve Bayes.

\section{METHODOLOGY}

This section elaborated in detail the method used. Figure 1 showed the overall process methodology in this study. The method can be divided into four sections specifically data acquisition, data pre-processing followed by feature extraction and finally gait classification.

\subsection{Data Acquisition}

In this study, data acquisition consists of 30 typically healthy children (TD) and 23 autistic children (ASD) with their ages ranging between 5 to 12 years old. Data acquisition experimental set-up is conducted in the Human Motion and Gait Analysis (HMGA) Premier Laboratory of Universiti Teknologi MARA (UiTM), Shah Alam, Selangor. Prior to this study, parents were given an information sheet that needs to be completed in the Consent Form based on recommendation and approval by UiTM Shah Alam Ethics Committee.

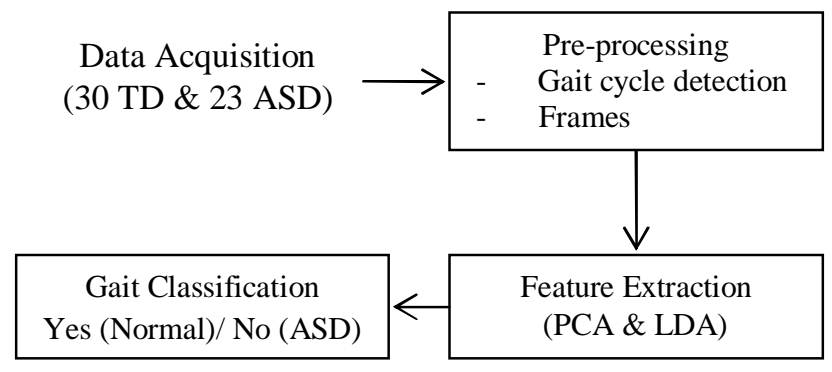

Figure 1: Overall Process Methodology

During data acquisition and experimental set-up, a depth camera is used as a motion sensing input device for the proposed markerless-based gait technique. This sensor provides the coordination of 20 primary body points as shown in Figure 2. The sensor is placed on a stand located 0.5 meter height from the floor and facing the subjects' walking direction. In addition, subjects can perform several walking trials for them to be comfortable and familiar with the experimental environment prior to data acquisition process. For the walking task, subjects are advised to walk freely bare footed with their comfortable speed to keep the nature in their walking behavior and without any assistant or walking aid. During experimental of data acquisition, the walking session is carried out few times and for each subject, at least ten successful walking trials are captured. As for the ASD group, as agreed by subjects and guardians, data collection for ASD children are acquired more than ten walking trials in ensuring optimum walking trials of ASD group are well captured.

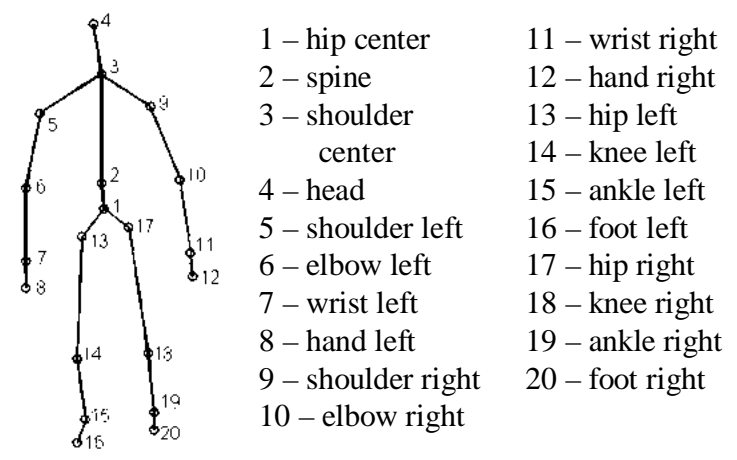

Figure 2: Twenty Skeleton Joint Point

\subsection{Data Pre-processing}

As for data pre-processing, firstly an empty frame from each trial was removed. Next, a customized function is used to detect and compute the gait cycle based on the distance between the left and right ankle [29], [31]. Further, the gait cycle is assessed using three consecutive local maxima of the calculated distance [32]. In this study, only one gait cycle is extracted from each trial. Due to variations of walking speeds among subjects, the number of extracted frames varies for 
each trial per subject. Also, in order to standardize the number of frames, the extracted walking trials are normalized using interpolation technique to disentangle the inconsistency that was based on 30 frames [33], [34]. Figure 3a showed the plot of the customized function for calculating the gait cycle, which is the distance from left to right ankles. The red dashed line represented one gait cycle namely three consecutive local maxima. Meanwhile, Figure $3 b$ depicted plots of frames before and after synchronization process that is fixed at 30 frames for each subject.
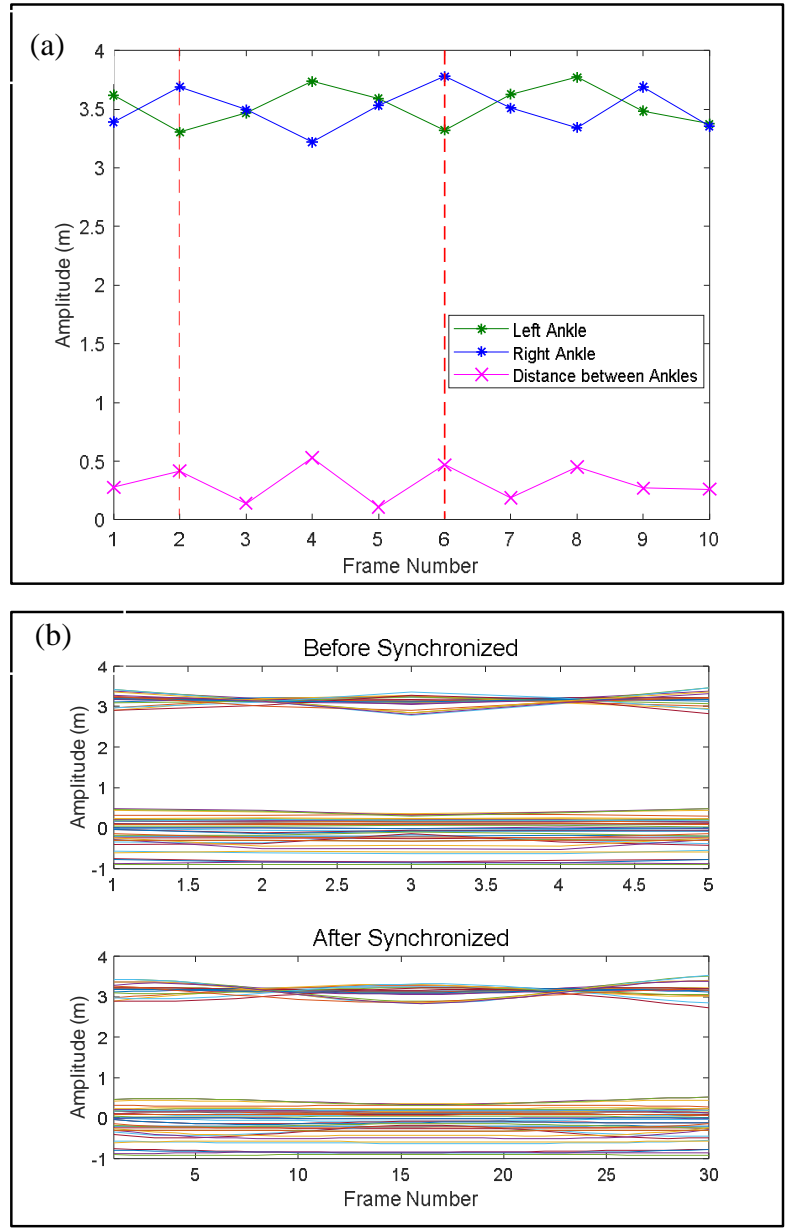

Figure 3: (a) Gait cycle detection using left and right ankle distance followed by three consecutive local maxima of the calculated distance; (b) Results of frame normalization based on 30 frames.

As mentioned earlier, the proposed markerless-based skeletal data is obtained using depth camera and motion sensor device. Recall that this sensor generated the 20 body points at $\mathrm{x}, \mathrm{y}$, and $\mathrm{z}$ coordinate or also known as the three dimensional (3D) data from the sensor position. As a result, a dataset with 60 attributes is derived from the 20 body points for each subject. The total gait features for each subject can be detailed as below:

- 20 skeletal points is multiplied by 3 xyz coordinates $=$ 60 features;
- Next, 60 features is multiplied by 30 frames $=1800$ gait features for each subject;

Further, for both TD and ASD group, the total features comprised of 1800 gait features and since each group has 300 walking trials, therefore the dimension of the extracted features for each group are 1800 by 300 . As depicted in Figure 4, this set of joint features is labeled and named as Direct Joint Feature (DIR) that can be defined as the features extracted by selecting all joints points in $\mathrm{x}, \mathrm{y}, \mathrm{z}$, coordinate. Next is the Reference Joint Feature (REF) and these features are obtained from the calculated distance between the reference joint which is the hip centre joint at point 1 to another body points specifically point 5 to point 20 . Hence, REF comprised of 480 feature size by 300 walking trials too. Further, both DIR and REF datasets will undergo feature extraction process.
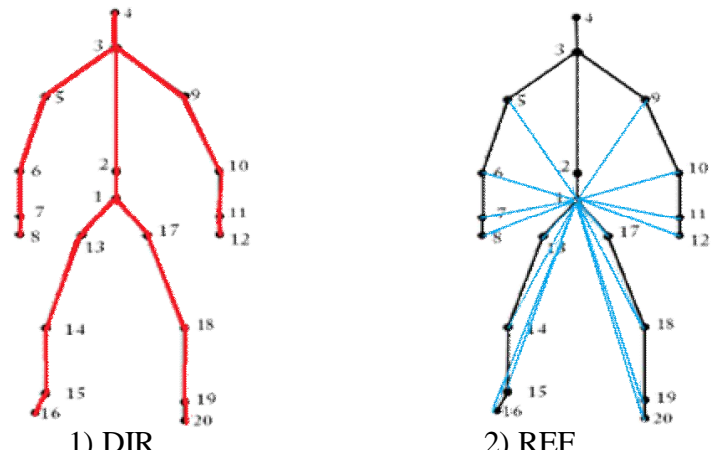

Figure 4: Illustration of DIR and REF Dataset

\subsection{Feature Extraction}

Recall that PCA and LDA are used as feature extraction in this study in extracting the significant gait features from the high dimensionality data. By applying these techniques, the original high dimensional gait features will be reduced with only the significant features to be used as inputs during classification. Next, the DIR and REF features sets are further optimized using PCA and LDA.

\section{A. Principal Component Analysis (PCA)}

PCA is a data representation method that can transform data into a new data space called principal components (PCs). This is achieved by calculating the eigenvalue or the singular value decomposition (SVD) of a data matrix [35]. The new set of dimensions or PCs are orthogonal in each other and ranked according to the variance of the data along them where the PC that has the largest variance occurs first. In this PCA implementation, three criteria are used to determine the significant $\mathrm{PC}(\mathrm{s})$. The $\mathrm{PC}$ is chosen based on the eigenvalue-one criteria (EOC), the cumulative percent of variance (CPV) and the scree plot method. Note that: 
- the EOC preserves components that owned eigenvalue equal or greater than unity (1);

- the CPV is based on PCs being employed as the components to obtain cumulative PV for at least $95 \%$.

- the scree plot is the representation of eigenvalues versus each component (PC). In the scree plot, PCs with large gap are retained and components with small gap are eliminated.

\section{B. Linear Discriminant Analysis (LDA)}

Next, LDA is evaluated as feature extraction in reducing the number of dimensions of original data of p-dimensional sample onto a smaller subspace. Unlike PCA, LDA attempts to find the feature subspace that enhances class separability (maximize class discrimination) and to serve this purpose it requires the class labels. Thus, by calculating the scatter matrices within the class $\left(\mathrm{S}_{\mathrm{W}}\right)$ and between the classes $\left(S_{B}\right)$, the generalized eigenvalues of the scatter matrices are computed. Further, to determine the linear discriminants for the new feature subspace, the eigenvectors are sorted in decreasing format. Here, the variance is calculated as the percentage of maximum discrimination since the linear combination required the largest mean difference between classes. Therefore, larger variance is expected to provide better separation, which implies the gap between the classes is high.

\subsection{Gait Classifier}

As stated earlier, the purpose of this study is to evaluate and validate the effectiveness of PCA and LDA as feature extraction. Thus, to estimate the generalization error of each classifier, the training data set is resampled using 10-fold cross-validation method. The effectiveness of both feature extraction is evaluated using three classifiers namely ANN, SVM and NB classifiers. Meanwhile for the output, each classifier is set as ' 0 ' for ASD and ' 1 ' for normal group.

\section{A. Artificial Neural Network (ANN)}

In this study, ANN is chosen as one of the classifiers. The architecture used consists of three layers that are the input layer, hidden layer and output layer. Next, multilayer perceptron (MLP) that utilized supervised learning technique called back propagation is chosen. The signals propagate forward from input to output. The inputs receive the signal and distribute it to every node in the hidden layer [14], [20] \& [22]. Every node in the hidden layer triggers, transform and pass the data to the output layer. Weights are defined for each connection through initialization function. A training error is calculated using the difference of the target response and the actual response. This error is fed back to the network and the network iteratively adjusts the different parameters of the network using learning algorithm until the desired output is acceptable. The process is repeated until it reaches the desired output. In this study, weights are adjusted by employing the Scaled Gradient Conjugate training algorithm. The tan-sigmod (tansig) transfer function is applied at the hidden and output layer. The optimize architecture of ANN is found to be at hidden neurons in hidden layer evaluated from 1 to 100 with increment of 10 .

\section{B. Support Vector Machine (SVM)}

The second classifier to be evaluated is the SVM that classifies data by finding the best hyperplane. The hyperplane is the plane that separates all data points of one class from the other class. The best hyperplane for SVM is one with the largest margin between the two classes [36]. In this study, three types of kernel functions namely linear, radial basis function $(\mathrm{RBF})$ and polynomial are used with the optimization of the regularization parameter; $C$ is set to $0.001,0.01,0.1,1$, 10, 100 and 1000 for each kernel. Meanwhile for SVM with polynomial kernel, an additional parameter is tested with three different degree of polynomial, $d p$ where $d p$ is set to 2, 3 and 4. For SVM with RBF kernel, the parameter tested is the value of gamma $(\gamma)$ set as $0.1,1$ and 10. Table 1 tabulated the kernel functions for the SVM classifier that is used to compute the element of $x_{j}$ and $x_{k}$ in $V\left(x_{j}, x_{k}\right)$ where $j$ and $k$ is the observations in predictor data, $x$.

Table 1: SVM Kernel Functions and Its Mathematical Equation

\begin{tabular}{|l|l|}
\hline $\begin{array}{l}\text { Kernel } \\
\text { function }\end{array}$ & \multicolumn{1}{c|}{ Mathematical formula } \\
\hline Linear & $V\left(x_{j}, x_{k}\right)=x_{j}^{\prime} x_{k}$ \\
\hline RBF & $V\left(x_{j}, x_{k}\right)=\exp \left(-\left\|x_{j}-x_{k}\right\|^{2}\right)$ \\
\hline Polynomial & $\begin{array}{l}V\left(x_{j}, x_{k}\right)=\left(1+x_{j}^{\prime} x_{k}\right)^{d p} \\
\text { where } d p \text { is the polynomial order. }\end{array}$ \\
\hline
\end{tabular}

\section{Nä̈ve Bayes (NB) Classifier}

Naive Bayes (NB) is a classification algorithm that assumes the presence of a feature in a class is unrelated to the presence of any other feature. NB classifies new data based on the highest probability of its belonging to a particular class $P(X \mid Y)$, given the densities, $P$ of the predictors, $X$ within each class, $Y$ [37]. In this study, two types of probability distribution are used that is normal or Gaussian distribution and kernel density estimation. In normal distribution, the distribution is suitable for predictors that have normal distributions in each class. Meanwhile for kernel distribution, it is suitable to be used for predictors with continuous distribution.

\section{RESULTS AND DISCUSSION}

This section will discuss the results attained using PCA and LDA as feature extraction followed by classification. Note that upon frame normalization, 30 frames are set for each walking trial per subject. These attributes are then arranged vertically concatenated via frame by frame so that the extracted features for each trial owned the dimension of 1800 by 1 per trial. In total, for DIR feature dataset, the feature size of DIR feature is 1800 by 300 whilst for REF, the feature size is 480 by 300 , with the row representing the number of 
attributes and the column representing the observations. Further, Table 2 tabulated the number of PCs and LDs retained upon completion of feature extraction. Based on criteria of EOC, the number of eigenvalue greater or equal than 1 are to be retained and the number of PCs that satisfied the criteria is the first five PCs for DIR feature dataset and only the first PC for REF feature dataset.

Table 2: Number of PCs to be retained based on results of PCA-EOC

\begin{tabular}{|c|c|c|}
\hline PC Number & DIR & REF \\
\hline 1 & 133.70 & 1.16 \\
\hline 2 & 8.71 & 0.26 \\
\hline 3 & 6.79 & 0.13 \\
\hline 4 & 3.52 & 0.08 \\
\hline 5 & 2.41 & 0.06 \\
\hline 6 & 0.55 & 0.04 \\
\hline
\end{tabular}

On the other hand, as depicted in Figure 5, the CPV is computed based on the cumulative variance for at least by 95\%. Hence, with CPV criteria, it was found that the first four PCs are to be retained for DIR feature dataset and 21 PCs for REF feature dataset. Next, as depicted in Figure 6 for the Scree Plot, the eigenvalues and the PCs are plotted for the Scree Test and the change of the slope is observed in order to choose the number of PCs. Results showed that four PCs are to be kept for DIR feature dataset whilst for REF dataset is three PCs.
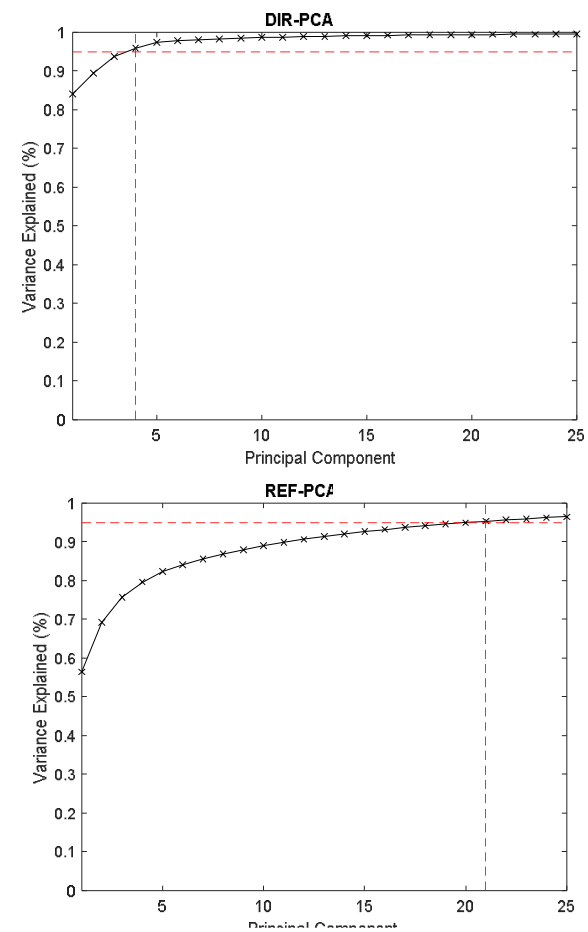

Figure 5: The CPV for DIR and REF dataset at $95 \%$ criterion

Meanwhile for LDA, it is found that the number of selected linear discriminant is 2 for both DIR and REF feature dataset respectively. The results of the first two LDs of LDA for DIR and REF are as shown in Figure 7. In addition, the overall results attained based on both PCA and LDA is as tabulated in Table 3. Note that both are linear transformation technique in reducing the dimensionality of the dataset. The difference is one is an unsupervised algorithm namely LDA since it ignores class labels in determining the directions or projections of the data that maximize the variance in a dataset, while the other one is a supervised algorithm that computes the directions (linear discriminants) of the data that maximize the separation between multiple classes.

Table 3: Number of significant features to be retained using PCA and LDA

\begin{tabular}{|l|c|c|}
\hline Feature Extraction Method & DIR & REF \\
\hline PCA-EOC & 5 & 1 \\
\hline PCA-CPV & 4 & 21 \\
\hline PCA-Scree Plot & 4 & 3 \\
\hline LDA & 2 & 2 \\
\hline
\end{tabular}
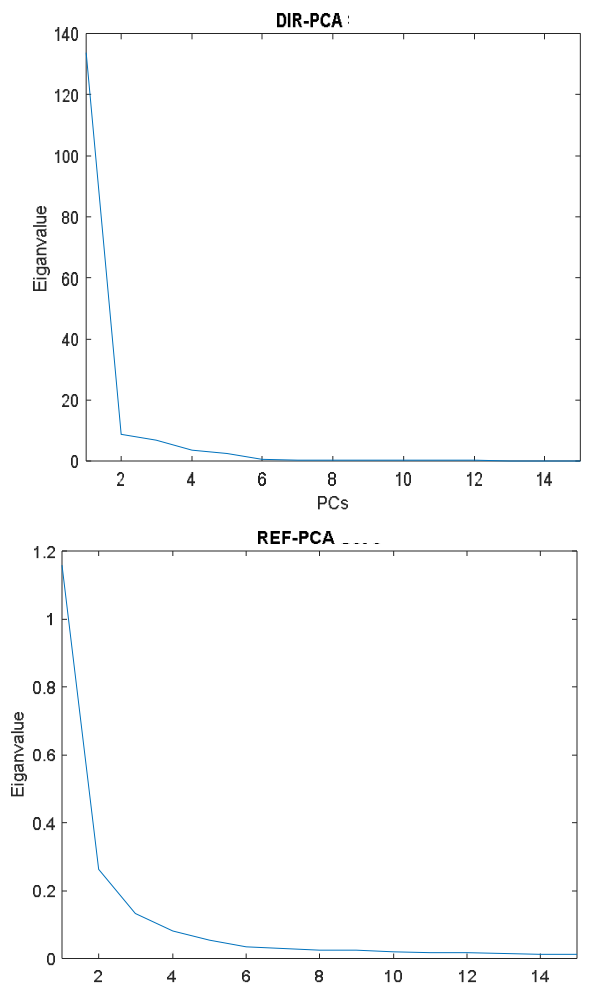

Figure 6: Scree Plot for DIR and REF dataset

Next is the classification phase. The extracted features namely DIR PCA-EOC, DIR PCA-CPV, DIR Scree, DIR LDA, REF PCA-EOC, REF PCA-CPV, REF Scree and REF LDA acted as input features to the classifiers. The 10-fold cross-validation method is used to validate the performances of the classifiers. Exhaustive evaluations of the highest accuracy for each classifier using these eight features are performed using a straightforward grid search approach with 10-fold cross-validation method for each DIR and REF feature dataset namely PCA-EOC, PCA-CPV, PCA-Scree and LDA as feature extraction. 

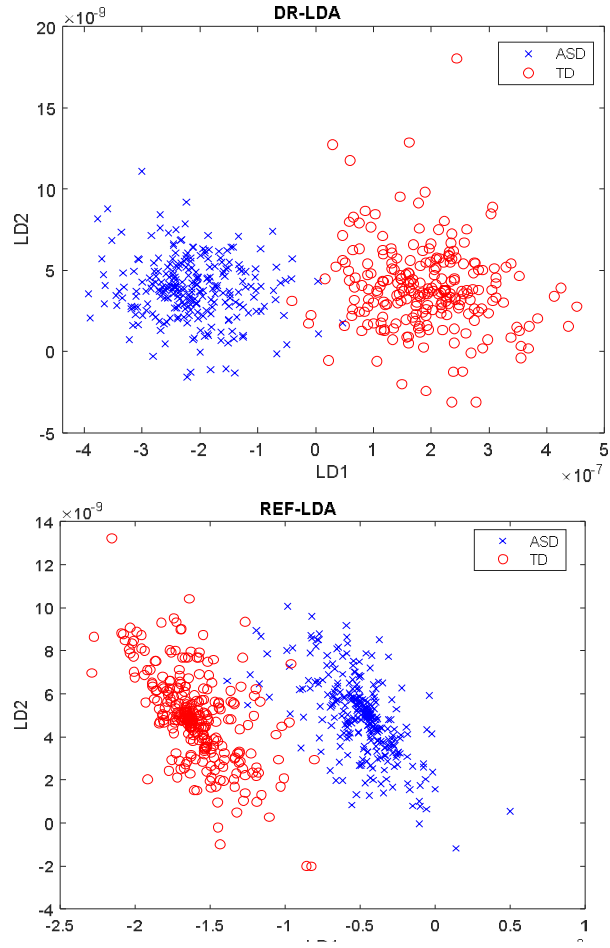

Figure 7: Plots of LDA based on LD1 and LD2 using DIR and REF Dataset

As depicted in Figure 8, excellent generalization performances are observed using features extracted using LDA as classifier inputs. Firstly, for DIR feature dataset, the highest accuracy rate achieved is NB classifier with $99.33 \%$ followed by ANN classifier at $99.30 \%$. As for REF feature dataset, the utmost accuracy rate is achieved using ANN classifier with $98.75 \%$, followed by SVM classifier with RBF kernel at a lower accuracy that is $98.67 \%$. Additionally, it is observed that the trends for both DIR and REF are similar for all classifiers. For DIR, the highest accuracy is using LDA followed by the PCA-EOC, PCA-CPV and PCA-Scree. Meanwhile for REF, once again the highest accuracy is based on LDA, followed by PCA-CPV, however PCA-Scree achieved better accuracy as compared to PCA-EOC. Here, it is shown that LDA as supervised algorithm performed well in discriminating the ASD and TD group based on both DIR and REF feature dataset. However, PCA-EOC for REF showed poor accuracy specifically below $65 \%$ for all three classifiers. Further, with LDA as the highest accuracy performance, Table 4 tabulated the sensitivity and specificity based on LDA and NB classifier since NB showed the highest accuracy amongst all three classifiers namely $99.33 \%$ using DIR dataset. From Table 4, as for sensitivity, this feature set is capable to classify the ASD group with sensitivity of $99.66 \%$
Accuracy (\%) using DIR feature dataset for SVM, NB \& ANN Classifiers
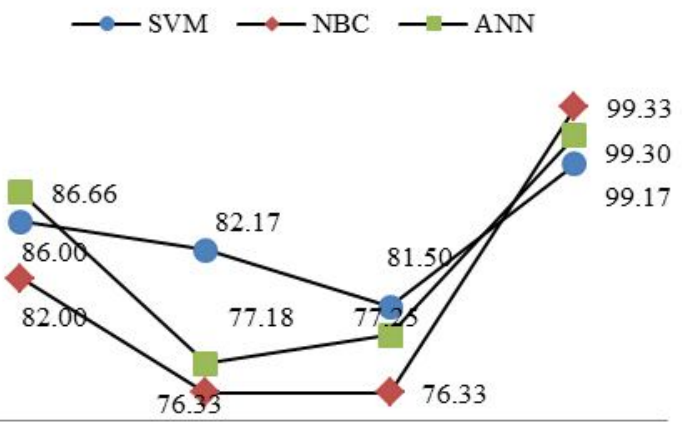

PCA-EOC PCA-CPV PCA-Scree LDA

\section{Accuracy (\%) using REF feature dataset for SVM, NB \& ANN Classifiers}
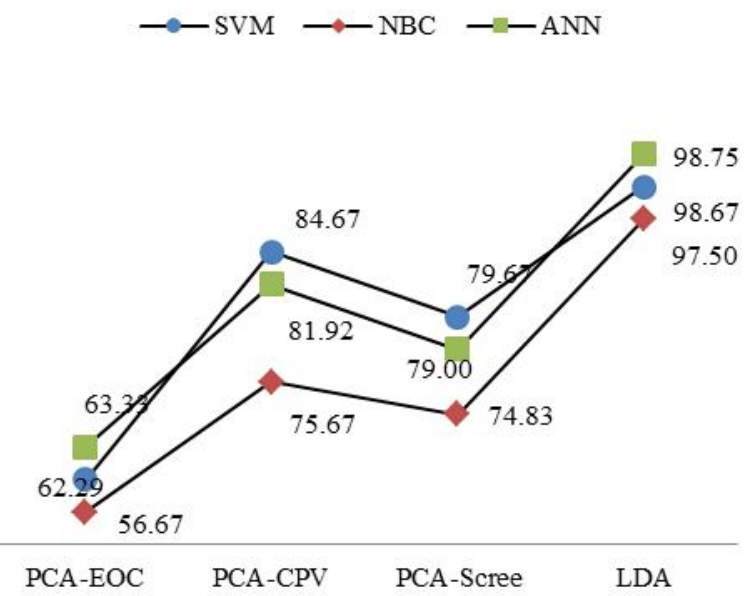

Figure 8: Classification Performance for all three classifiers (SVM, NB and ANN) using DIR and REF dataset

Table 4: Performance Measure (\%) for all three classifiers using LDA based on DIR and REF dataset

\begin{tabular}{|c|c|c|c|c|c|c|}
\hline \multirow{2}{*}{ 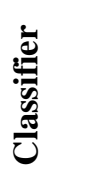 } & \multicolumn{2}{|c|}{ Acc } & \multicolumn{2}{|c|}{ Sens } & \multicolumn{2}{|c|}{ Spec } \\
\hline & DIR & REF & DIR & REF & DIR & REF \\
\hline SVM & 99.27 & 98.67 & 99.66 & 98.99 & 98.68 & 98.34 \\
\hline NBC & 99.33 & 97.59 & 99.66 & 98.30 & 99.00 & 96.72 \\
\hline ANN & 99.30 & 98.75 & 99.49 & 98.63 & 99.00 & 98.86 \\
\hline
\end{tabular}

whilst the TD group with specificity of $99.00 \%$. On the other hand, the least accuracy (Acc) found is the SVM classifier with $98.67 \%$ for REF dataset that correctly classify the ASD group with a sensitivity (Sens) of $98.99 \%$ and TD group with the specificity (Spec) of $98.34 \%$ only as compared to NB classifier. Therefore, between the two features and as for feature extraction, DIR-LDA is the most apposite for ASD gait classification based on this proposed markerless-based gait features. 


\section{CONCLUSION}

In conclusion, it was proven that LDA is better than PCA as feature extraction based on the performance of classification with NB classifier surpassed SVM and ANN classifiers. In addition, amongst the two features namely DIR and REF gait features, DIR feature dataset demonstrated its suitability for ASD gait classification for this proposed markerless-based model. Future work includes implementation on other markerless-based feature such as depth images. It is hoped that findings from this study could assist both medical practitioner and physiotherapist in planning suitable gait analysis intervention program for autistic children.

\section{ACKNOWLEDGEMENT}

This research is funded by Research Management Centre (RMC), Universiti Teknologi MARA (UiTM), Shah Alam, Selangor, Malaysia Grant No: 600-IRMI/MyRA5/3/BESTARI (041/2017) and 600-IRMI 5/3/GIP (084/2018). The authors would like to thank UiTM for the facilities provided for this research namely the Human Motion and Gait Analysis Premier Laboratory and the National Autism Society of Malaysia (NASOM) for the volunteered participants and guidance.

\section{REFERENCES}

1. A. P. Association, Diagnostic and statistical manual of mental disorders (DSM-5®): American Psychiatric Pub, 2013.

2. M. Nobile, P. Perego, L. Piccinini, E. Mani, A. Rossi, M. Bellina, and M. Molteni, "Further evidence of complex motor dysfunction in drug naïve children with autism using automatic motion analysis of gait," Autism, vol. 15, pp. 263-283, 2011.

https://doi.org/10.1177/1362361309356929

3. G. Esposito and P. Venuti, "Analysis of Toddlers' Gait after Six Months of Independent Walking to Identify Autism: A Preliminary Study 1," Perceptual and Motor Skills, vol. 106, pp. 259-269, 2008.

https://doi.org/10.2466/pms.106.1.259-269

4. S. J. Spence, P. Sharifi, and M. Wiznitzer, "Autism spectrum disorder: screening, diagnosis, and medical evaluation," in Seminars in Pediatric Neurology, 2004, pp. 186-195.

5. E. I. de Bruin, R. Blom, F. M. Smit, F. J. van Steensel, and S. M. Bögels, "MYmind: Mindfulness training for youngsters with autism spectrum disorders and their parents," Autism, vol. 19, pp. 906-914, 2015.

6. J. D. Eggleston, J. R. Harry, and J. S. Dufek, "Lower extremity joint stiffness during walking distinguishes children with and without autism," Human Movement Science, vol. 62, pp. 25-33, 2018.

https://doi.org/10.1016/j.humov.2018.09.003

7. J. S. Dufek, J. R. Harry, J. D. Eggleston, and R. A. Hickman, "Walking Mechanics and Movement Pattern Variability in Monozygotic Twins with
Autism Spectrum Disorder," Journal of Developmental and Physical Disabilities, vol. 30, pp. 793-805, 2018.

8. C. Hasan, R. Jailani, N. M. Tahir, and H. Desa, "Vertical ground reaction force gait patterns during walking in children with autism spectrum disorders," Int. J. Eng. Trans. B Appl., vol. 31, pp. 705-711, 2018. https://doi.org/10.5829/ije.2018.31.05b.04

9. C.-S. Yang, G.-S. Lee, Y.-T. Lim, and B.-O. Lim, "Comparative Gait Analysis Between Children with Autism and Age Matched Controls," in ISBS-Conference Proceedings Archive, 2014.

10. M. M. Nor, R. Jailani, N. Tahir, I. M. Yassin, Z. I. Rizman, and R. Hidayat, "EMG signals analysis of BF and RF muscles in autism spectrum disorder (ASD) during walking," International Journal on Advanced Science, Engineering and Information Technology, vol. 6, pp. 793-798, 2016.

11. B.-O. Lim, D. O'Sullivan, B.-G. Choi, and M.-Y. Kim, "Comparative gait analysis between children with autism and age-matched controls: analysis with temporal-spatial and foot pressure variables," Journal of Physical Therapy Science, vol. 28, pp. 286-292, 2016. https://doi.org/10.1589/jpts.28.286

12. C.-S. Yang, G.-S. Lee, B.-K. Choi, D. O'Sullivan, Y.-H. Kwon, and B.-O. Lim, "Gait Analysis in Children with Autism using Temporal-Spatial and Foot Pressure Variables," in ISBS-Conference Proceedings Archive, 2012, pp. 307-310.

13. J. A. Vilensky, A. R. Damasio, and R. G. Maurer, "Gait disturbances in patients with autistic behavior: a preliminary study," Archives of Neurology, vol. 38, pp. 646-649, 1981.

14. S. Ilias, N. M. Tahir, R. Jailani, and C. Z. C. Hasan, "Classification of autism children gait patterns using Neural Network and Support Vector Machine," in Computer Applications \& Industrial Electronics (ISCAIE), 2016 IEEE Symposium on, 2016, pp. 52-56.

15. C. Z. C. Hasan, R. Jailani, and N. M. Tahir, "ANN and SVM Classifiers in Identifying Autism Spectrum Disorder Gait Based on Three-Dimensional Ground Reaction Forces," in TENCON 2018-2018 IEEE Region 10 Conference, 2018, pp. 2436-2440.

16. M. Calhoun, M. Longworth, and V. L. Chester, "Gait patterns in children with autism," Clinical Biomechanics, vol. 26, pp. 200-206, 2011.

17. [M. Z. Al-Faiz and Z. J.Ali, "Humanoid Robotic Hand (HRH) Based on EMG signal for Amputees Persons," International Journal of Emerging Trends in Engineering Research, vol. 6, pp. 19-26, 2018. https://doi.org/10.30534/ijeter/2018/02642018

18. D. Slijepcevic, M. Zeppelzauer, C. Schwab, A.-M. Raberger, C. Breiteneder, and B. Horsak, "Input representations and classification strategies for automated human gait analysis," Gait \& posture, vol. 76, pp. 198-203, 2020. https://doi.org/10.1016/j.gaitpost.2019.10.021

19. X. Hongye and H. Zhuoya, "Gait recognition based on gait energy image and linear discriminant analysis," in 2015 IEEE International Conference on Signal 
Processing, Communications and Computing (ICSPCC), 2015, pp. 1-4.

20. S. Wen, F. Wang, and C. Wu, "Realtime gait kinematics classification using LDA and SVM," in 2011 Chinese Control and Decision Conference (CCDC), 2011, pp. 592-595.

21. H. H. Manap, N. M. Tahir, A. I. M. Yassin, and R. Abdullah, "Anomaly gait classification of Parkinson disease based on ANN," in 2011 IEEE International Conference on System Engineering and Technology, 2011, pp. 5-9.

22. R. Subba and A. K. Bhoi, "Feature Extraction and Classification Between Control and Parkinson's Using EMG Signal," in Cognitive Informatics and Soft Computing, ed: Springer, 2020, pp. 45-52. https://doi.org/10.1007/978-981-15-1451-7_5

23. K. Kaczmarczyk, A. Wit, M. Krawczyk, and J. Zaborski, "Gait classification in post-stroke patients using artificial neural networks," Gait \& posture, vol. 30, pp. 207-210, 2009.

24. B.-1. Zhang, Y. Zhang, and R. K. Begg, "Gait classification in children with cerebral palsy by Bayesian approach," Pattern Recognition, vol. 42, pp. 581-586, 2009.

25. Y. Zhou, R. Romijnders, C. Hansen, J. van Campen, W. Maetzler, T. Hortobágyi, and C. J. Lamoth, "The detection of age groups by dynamic gait outcomes using machine learning approaches," Scientific Reports, vol. 10, pp. 1-12, 2020. https://doi.org/10.1038/s41598-020-61423-2

26. B. M. Eskofier, P. Federolf, P. F. Kugler, and B. M. Nigg, "Marker-based classification of young-elderly gait pattern differences via direct PCA feature extraction and SVMs," Computer methods in biomechanics and biomedical engineering, vol. 16, pp. 435-442, 2013.

27. A. S. Ashour, A. El-Attar, N. Dey, H. A. El-Kader, and M. M. A. El-Naby, "Long short term memory based patient-dependent model for FOG detection in Parkinson's disease," Pattern Recognition Letters, vol. 131, pp. 23-29, 2020.

28. J. Sutopo, M. K. A. Ghani, M. A. Burhanuddin, and Zulhawati, "Gesture Recognition of Dance using Chain Code and Hidden Markov Model," International Journal of Advanced Trends in Computer Science and Engineering, vol. 8(6), pp. 3194-3199, 2019. https://doi.org/10.30534/ijatcse/2019/85862019

29. R. Sahak, N. M. Tahir, A. I. Yassin, F. K. Zaman, and A. Zabidi, "Frontal view gait recognition using locally linear embedded and multilayer perceptron based on Kinect," in Signal Processing \& its Applications (CSPA), 2017 IEEE 13th International Colloquium on, 2017, pp. 303-308.

30. I. Sreenidhi and P. Satyanarayana, "Real-Time Human Fall Detection and Emotion Recognition using Embedded Device and Deep Learning," International Journal of Emerging Trends in Engineering Research, vol. 8, pp. 780-786, 2020. https://doi.org/10.30534/ijeter/2020/28832020

31. F. Ahmed, P. Polash Paul, and M. L. Gavrilova, "Kinect-based gait recognition using sequences of the most relevant joint relative angles," Journal of WSCG, vol. 23, pp. 147-156, 2015.

32. W. Kim and Y. Kim, "Gait Recognition for Human Identification using Kinect," in Proceedings of the International Conference on Research in Adaptive and Convergent Systems, 2017, pp. 1-4.

33. E. Ghorbel, R. Boutteau, J. Boonaert, X. Savatier, and S. Lecoeuche, "3D real-time human action recognition using a spline interpolation approach," in 2015 International Conference on Image Processing Theory, Tools and Applications (IPTA), 2015, pp. 61-66.

34. F. Sun, W. Zang, R. Gravina, G. Fortino, and Y. Li, "Gait-based identification for elderly users in wearable healthcare systems," Information fusion, vol. 53, pp. 134-144, 2020.

35. M. Ringnér, "What is principal component analysis?," Nature biotechnology, vol. 26, p. 303, 2008.

36. C. Cortes and V. Vapnik, "Support-vector networks," Machine learning, vol. 20, pp. 273-297, 1995. https://doi.org/10.1007/BF00994018

37. C. Borgelt and J. Gebhardt, "A naive bayes style possibilistic classifier," in Proceedings of 7th European Congress on Intelligent Techniques and Soft Computing (EUFIT'99), 1999. 